\title{
Local Properties of Measures in Quantum Field Theory and Cosmology
}

\author{
José M. VELHINHO \\ Faculdade de Ciências, Universidade da Beira Interior, \\ R. Marquês D'Ávila e Bolama, 6201-001 Covilhã, Portugal \\ E-mail: jvelhi@ubi.pt
}

Received October 30, 2014, in final form January 14, 2015; Published online January 17, 2015

http://dx.doi.org/10.3842/SIGMA.2015.006

\begin{abstract}
We show that measure theoretical results concerning the Ashtekar-Lewandowski measure in the space of generalized connections have direct analogues in the context of the Bohr compactification of the line and associated Haar measure. We present also a characterization of the support of the measure associated with the canonical quantization of the free massive scalar field, following closely well known analogous results concerning the Euclidean path integral measure.
\end{abstract}

Key words: canonical quantization; scalar field; loop cosmology; support of the measure

2010 Mathematics Subject Classification: 28C10; 28C20; 81T99; 83F99

\section{Introduction}

Measures in infinite-dimensional spaces, linear and otherwise, feature prominently in quantum theory. One can find them in the quantum theory of scalar fields, both from the Euclidean path integral and from the canonical quantization perspectives (see, e.g., [9]), in the quantization of gravity (see $[2,14]$ ) and in quantum cosmology $[1,3]$. Typically, these measures are defined on extensions of the spaces associated with the corresponding field theories or classical mechanical systems. In the quantum theory of scalar fields one finds measures in the space $\mathcal{S}^{\prime}\left(\mathbb{R}^{d}\right)$ of tempered distributions, dual to the space of Schwartz functions. In quantum gravity, in the socalled loop approach, one finds measures in the space of generalized connections $[2,10,14,16]$, which is a natural (though quite large) extension of the space of connections on a manifold. In both cases the distributional extensions are crucial, given that, for measures of interest, the classical non-distributional configurations corresponding to standard functions or connections turn out to be irrelevant sets, i.e. subsets of zero measure sets. A similar situation appears in the loop approach to quantum cosmology $[1,3,4,5]$. In this case a role analogous to that of the space of generalized connections is played by the Bohr compactification of the line, a well know compact extension of $\mathbb{R}$.

In the present paper we characterize the support of two different measures of interest, in terms of local properties. First, following closely [12] and [8], we consider sets of measure zero and of measure one for the Gaussian measure in $\mathcal{S}^{\prime}\left(\mathbb{R}^{d}\right)$ of covariance

$$
\langle\phi(f), \phi(g)\rangle=\frac{1}{2} \int d^{d} x f\left(m^{2}-\Delta\right)^{-1 / 2} g .
$$

This is the measure uniquely associated with the free scalar field of mass $m$, in $d$-spatial dimensions, in the canonical quantization formalism (see [9]), rather than the Euclidean path integral measure for $d$-spacetime dimensions considered in $[8,12]$. Second, we show that measure theoretical results proved in [11], concerning the so-called Ashtekar-Lewandowski measure in the space of generalized connections, have close analogues in the context of the Bohr compactification of $\mathbb{R}$ 
and associated Haar measure. In particular, we give a local characterization of the support of this Haar measure and display an ergodic action which in some sense mimics the action of spatial diffeomorphisms in the space of generalized connections.

\section{Canonical free field measure}

Consider the linear space of real Schwartz functions $\mathcal{S}\left(\mathbb{R}^{d}\right)$, with its standard nuclear topology, and let $\mathcal{S}^{\prime}\left(\mathbb{R}^{d}\right)$ denote its topological dual. As is well known, a scalar product $($,$) on \mathcal{S}\left(\mathbb{R}^{d}\right)$, continuous with respect to the nuclear topology, defines a Gaussian measure on $\mathcal{S}^{\prime}\left(\mathbb{R}^{d}\right)$, of mean zero and covariance $\langle\phi(f), \phi(g)\rangle=(f, g)$. (We will use the term covariance to denote also the scalar product $($, ) that defines the measure.) A refinement of this result is given by Minlos' theorem for the case of Gaussian measures, which can be stated as follows (see $[12,13,18]$ ):

Theorem 1 (Minlos). Let $E$ be a real nuclear space, $($,$) a continuous scalar product and \mathcal{H}$ the completion of $E$ with respect to $($,$) . Let H$ be an injective Hilbert-Schmidt operator on $\mathcal{H}$, such that $E \subset H \mathcal{H}$ and $H^{-1}: E \rightarrow \mathcal{H}$ is a continuous map. Let $(,)_{1}$ be the scalar product on $E$ defined by $(f, g)_{1}=\left(H^{-1} f, H^{-1} g\right)$. Then, the Gaussian measure on the dual space $E^{\prime}$ with covariance $($,$) is supported on the subspace of E^{\prime}$ of those functionals which are continuous with respect to the topology defined by $(,)_{1}$.

Let us mention also a result by Colella and Lanford [8, Proposition 3.1], applicable to Gaussian measures in $\mathcal{S}^{\prime}\left(\mathbb{R}^{d}\right)$ which are invariant under $\mathbb{R}^{d}$ translations. Given a covariance $($,$) on \mathcal{S}\left(\mathbb{R}^{d}\right)$, its kernel $\mathcal{C}$ is the distribution on $\mathbb{R}^{2 d}$ defined by

$$
(f, g)=: \int d^{d} x d^{d} x^{\prime} f(x) \mathcal{C}\left(x, x^{\prime}\right) g\left(x^{\prime}\right), \quad \forall f, g \in \mathcal{S}\left(\mathbb{R}^{d}\right) .
$$

Clearly, the corresponding measure in $\mathcal{S}^{\prime}\left(\mathbb{R}^{d}\right)$ is invariant under $\mathbb{R}^{d}$ translations if and only if $\mathcal{C}\left(x, x^{\prime}\right)=\mathcal{C}\left(x-x^{\prime}\right)$. Let us recall that a distribution $\phi \in \mathcal{S}^{\prime}\left(\mathbb{R}^{d}\right)$ can be identified with a signed measure on a open set $U \subset \mathbb{R}^{d}$ if there is a measure $\nu$ on $U$ and an integrable function $F$ such that $\phi(f)=\int_{U} f F d \nu$, for every $f \in \mathcal{S}\left(\mathbb{R}^{d}\right)$ supported in $U$. Then [8]:

Theorem 2. Let $\mu$ be a Gaussian measure in $\mathcal{S}^{\prime}\left(\mathbb{R}^{d}\right)$, invariant with respect to $\mathbb{R}^{d}$ translations and such that the kernel $\mathcal{C}$ of the corresponding covariance is not a continuous function. Then, for $\mu$-almost every $\phi \in \mathcal{S}^{\prime}\left(\mathbb{R}^{d}\right)$ there is no non-empty open set $U \subset \mathbb{R}^{d}$ on which $\phi$ can be seen as a signed measure.

\subsection{Local support properties}

Let us then consider the measure on $\mathcal{S}^{\prime}\left(\mathbb{R}^{d}\right)$ which provides the representation of the canonical commutation relations associated with the free real scalar field of nonzero mass $m$, in $d$ spatial dimensions. This is the Gaussian measure of covariance

$$
(f, g)_{m}:=\frac{1}{2} \int d^{d} x f\left(m^{2}-\Delta\right)^{-1 / 2} g, \quad f, g \in \mathcal{S}\left(\mathbb{R}^{d}\right),
$$

where the mass is a real parameter and $\Delta$ is the Laplacian. (See [9] for the relation between the Euclidean path integral, or constructive, formulation and the canonical quantization approach.)

One can now apply the general results above to obtain both a nontrivial zero measure set and nontrivial sets of measure one, for the measure associated with the free field.

First, let us consider the kernel $\mathcal{C}_{m}$ of the covariance (1). It is clear that $\mathcal{C}_{m}\left(x, x^{\prime}\right)=\mathcal{C}_{m}\left(x-x^{\prime}\right)$, and the distribution $\mathcal{C}_{m}(x)$ is easily obtained, after Fourier transformation, as

$$
\mathcal{C}_{m}(x)=\frac{1}{(2 \pi)^{d}} \int d^{d} p \frac{e^{i p x}}{2\left(m^{2}+p^{2}\right)^{1 / 2}} .
$$


One can immediately conclude that, for any dimension $d(>0)$ and any value of the mass $m, \mathcal{C}_{m}(x)$ is not a continuous function, given that the Fourier transform $\left(m^{2}+p^{2}\right)^{-1 / 2}$ is not integrable, independently of $d$. So, by Theorem 2, it follows that the set of distributions which, even if locally, can be identified with signed measures, does not contribute to the measure defined by (1).

We will now apply Theorem 1 to the canonical free field measure defined by (1), in order to obtain sets of measure one. We follow here $[8,12,13]$, adapting the results therein to the canonical formalism. Let us show that the distributions that support the measure are such that the action of the operator $\left(1+x^{2}\right)^{-\alpha}\left(m^{2}-\Delta\right)^{-\beta}$ produces $L^{2}\left(\mathbb{R}^{d}\right)$ elements, for $\alpha>d / 4$ and $\beta>(d-1) / 4$. To see this, let us consider the operators

$$
\widetilde{H}:=\left(m^{2}-\Delta\right)^{-\alpha}\left(1+x^{2}\right)^{-\alpha}
$$

on $L^{2}\left(\mathbb{R}^{d}\right)$, where $\left(1+x^{2}\right)$ is a multiplication operator and $\alpha>d / 4$. Since $\left(1+x^{2}\right)^{-\alpha}$ is square integrable and the same is true for the Fourier transform $\left(m^{2}+p^{2}\right)^{-\alpha}$ of $\left(m^{2}-\Delta\right)^{-\alpha}$, the operators $\widetilde{H}$ are of the Hilbert-Schmidt type $\forall \alpha>d / 4$ (see [12, Lemma 1]). Let us introduce the Hilbert space $\mathcal{H}_{m}^{-}$, which is the completion of $\mathcal{S}\left(\mathbb{R}^{d}\right)$ with respect to the inner product (1). Using the unitary transformation

$$
\left(m^{2}-\Delta\right)^{1 / 4}: L^{2}\left(\mathbb{R}^{d}\right) \rightarrow \mathcal{H}_{m}^{-}
$$

we define the following Hilbert-Schmidt operator on $\mathcal{H}_{m}^{-}$:

$$
H=\left(m^{2}-\Delta\right)^{1 / 4} \widetilde{H}\left(m^{2}-\Delta\right)^{-1 / 4} .
$$

Let us finally introduce the scalar product in $\mathcal{S}\left(\mathbb{R}^{d}\right)$ :

$$
\begin{aligned}
(f, g)_{1} & :=\int d^{d} x\left(\left(m^{2}-\Delta\right)^{-1 / 4} H^{-1} f\right)\left(\left(m^{2}-\Delta\right)^{-1 / 4} H^{-1} g\right) \\
& =\int d^{d} x\left(\left(1+x^{2}\right)^{\alpha}\left(m^{2}-\Delta\right)^{\beta} f\right)\left(\left(1+x^{2}\right)^{\alpha}\left(m^{2}-\Delta\right)^{\beta} g\right),
\end{aligned}
$$

where $\beta=\alpha-1 / 4>(d-1) / 4$. By Theorem 1 , the subspace of those functionals which are continuous with respect to $(,)_{1}$ is a set of measure one. Invoking the Riesz lemma, for each such functional $\phi$ there is a unique element $f$ of the completion of $\mathcal{S}\left(\mathbb{R}^{d}\right)$ with respect to $(,)_{1}$ such that

$$
\phi(g)=(f, g)_{1}=\int d^{d} x\left(\left(m^{2}-\Delta\right)^{\beta}\left(1+x^{2}\right)^{\alpha}\left(1+x^{2}\right)^{\alpha}\left(m^{2}-\Delta\right)^{\beta} f\right) g .
$$

Since $\left(1+x^{2}\right)^{\alpha}\left(m^{2}-\Delta\right)^{\beta} f$ above belongs to $L^{2}\left(\mathbb{R}^{d}\right)$, one can say that continuous functionals are uniquely identified as elements of the form $\left(m^{2}-\Delta\right)^{\beta}\left(1+x^{2}\right)^{\alpha} \psi$, where $\psi \in L^{2}\left(\mathbb{R}^{d}\right)$ and those elements are seen as functionals by means of integration (or the $L^{2}\left(\mathbb{R}^{d}\right)$ inner product). The support of the measure can therefore be written as follows

$$
\left(m^{2}-\Delta\right)^{\beta}\left(1+x^{2}\right)^{\alpha} L^{2}\left(\mathbb{R}^{d}\right),
$$

in the previously announced sense that the distributions which support the measure are such that the application of the operator $\left(1+x^{2}\right)^{-\alpha}\left(m^{2}-\Delta\right)^{-\beta}$ produces elements of $L^{2}\left(\mathbb{R}^{d}\right)$, for $\alpha>d / 4$ and $\beta>(d-1) / 4$.

From a local point of view, applying the operator $\left(m^{2}-\Delta\right)^{-\beta}$ to a typical distribution is sufficient to produce a locally $L^{2}$ function. In other words, one can say that the Fourier transform of $\left(m^{2}+p^{2}\right)^{-\beta} \widetilde{\phi}(p)$ is locally $L^{2}$, for almost every distribution $\phi(x)$, where $\widetilde{\phi}(p)$ denotes the Fourier transform. In the most favourable case $d=1$, any negative power of $\left(m^{2}+p^{2}\right)$ achieves 
this effect. Further applying the operator $\left(1+x^{2}\right)^{-\alpha}$ regularizes the behaviour at infinity of typical distributions, producing $L^{2}$ elements.

Let us conclude this section with a couple of comments.

Note that although the mass $m$ appears explicitly in the characterization of the support (3), the space that one obtains for support of the measure as a consequence of Minlos' theorem is actually the same for all values of the mass. This can be seen, e.g., from the fact that the topology defined by the scalar product (2) is independent of the value of the mass (as long as it is not zero). So, the above characterization of the support is not sensitive to the value of the mass. This can be traced back to the fact that $\left(m^{2}-\Delta\right)^{-1 / 2}\left(m^{\prime 2}-\Delta\right)^{1 / 2}$ is a bounded operator, of bounded inverse, $\forall m, m^{\prime}$, and the situation should therefore not change with any other choice of Hilbert-Schmidt operator in Theorem 1.

Nevertheless, it is well known that the measures associated with two distinct values of the mass are in fact singular with respect to each other. Therefore, disjoint supports can be found, for distinct masses. To unveil these crucial differences in the support requires a different type of analysis of the large scale behaviour of typical distributions (see [11] for a detailed study).

Since the differences between the supports depend on the large scale, rather than on the local behaviour, the mutual singularity of distinct free field measures disappears when the space $\mathbb{R}^{d}$ is replaced by a compact manifold. From the measure theoretical perspective, this is at the root of the recent uniqueness of quantization results proved in [7] and references therein. In fact, mutual singularity of the measures translates into unitary inequivalence of the corresponding representations of the canonical commutation relations, which is at the heart of the obstruction to generalize the results of [7] to the noncompact case.

\section{Haar measure in the Bohr compactification of the line}

The second measure we wish to consider is the Haar measure on the Bohr compactification of the line. The space in question, which we will denote by $\overline{\mathbb{R}}$, can be seen as the set of all group morphisms from $\mathbb{R}$ to the unit circle $T$, with an appropriate topology.

This space arises naturally in the approach to quantum cosmology known as loop quantum cosmology $[1,3,4,5,6]$, where it plays a role analogous to that of the space of generalized connections in full blown loop quantum gravity. It should be said, however, that from a more physical viewpoint, the role of $\overline{\mathbb{R}}$ is less prominent than that of the space of generalized connections. In fact, although $\overline{\mathbb{R}}$ can be seen as the initial quantum configuration space for loop quantum cosmology $[1,17]$, subsequent developments lead to an effectively smaller space. The meaning of this is that whereas the quantum theory is initially constructed over the non-separable Hilbert space of square integrable functions on $\overline{\mathbb{R}}$, one ends up with a separable subspace thereof $[3,15]$. Also, in Bojowald's new quantization proposal [6] one already starts from a Hilbert space which is different from the space of square integrable functions on $\overline{\mathbb{R}}$.

Let us then consider the line $\mathbb{R}$ with its commutative group structure given by addition of real numbers. With the appropriate topology to be described below, the Bohr compactification $\overline{\mathbb{R}}$ is the set $\operatorname{Hom}[\mathbb{R}, T]$ of all, not necessarily continuous, group morphisms from $\mathbb{R}$ to the multiplicative group $T$ of unitaries in the complex plane:

$$
\overline{\mathbb{R}} \equiv \operatorname{Hom}[\mathbb{R}, T] .
$$

The generic element of $\overline{\mathbb{R}}$ is here denoted by $\bar{x}$. So, every $\bar{x} \in \overline{\mathbb{R}}$ is a map, $\bar{x}: \mathbb{R} \rightarrow T$ such that

$$
\bar{x}(0)=1 \quad \text { and } \quad \bar{x}\left(k_{1}+k_{2}\right)=\bar{x}\left(k_{1}\right) \bar{x}\left(k_{2}\right), \quad \forall k_{1}, k_{2} \in \mathbb{R} .
$$

Since $T$ is commutative, it is clear that

$$
\bar{x} \bar{x}^{\prime}(k):=\bar{x}(k) \bar{x}^{\prime}(k)
$$


defines a group structure on $\overline{\mathbb{R}}$, which is again commutative. Concerning the topology, note first that $\overline{\mathbb{R}}$ is a subgroup of the group of all (not necessarily morphisms) maps from $\mathbb{R}$ to $T$. Since the latter can be identified with the product group $\times_{k \in \mathbb{R}} T$, and $T$ is compact, it carries the Tychonoff product topology. The product $\times_{k \in \mathbb{R}} T$ thus becomes a compact (Hausdorff) group. This topology descends to $\overline{\mathbb{R}}$, making it a topological group. Finally, from the fact that $\overline{\mathbb{R}}$ contains only morphisms, one can easily check that it is a closed subset of $\times_{k \in \mathbb{R}} T$, and it is therefore compact.

So, $\overline{\mathbb{R}}$ is a commutative compact group, with respect to the Tychonoff topology and the group operation (4). Because it is compact, $\overline{\mathbb{R}}$ is equipped with a normalized invariant (under the group operation) measure, namely the Haar measure, here denoted by $\mu_{0}$.

To proceed, it is convenient to introduce the projective structure of $\overline{\mathbb{R}}$, which we now review very briefly (see [17] for details).

For arbitrary $n \in \mathbb{N}$, let us consider finite sets $\gamma=\left\{k_{1}, \ldots, k_{n}\right\}$ of independent real numbers $k_{1}, \ldots, k_{n}$, with respect to the additive group operation in $\mathbb{R}$, i.e. such that the condition

$$
\sum_{i=1}^{n} m_{i} k_{i}=0, \quad m_{i} \in \mathbb{Z},
$$

can only be fulfilled with $m_{i}=0, \forall i$. (This notion of independence, used in [17], is of course readily seen to be the same as linear independence over $\mathbb{Q}$.) Let $G_{\gamma}$ denote the subgroup of $\mathbb{R}$ freely generated by the set $\gamma=\left\{k_{1}, \ldots, k_{n}\right\}$ :

$$
G_{\gamma}:=\left\{\sum_{i=1}^{n} m_{i} k_{i}, m_{i} \in \mathbb{Z}\right\} .
$$

For each independent set $\gamma$, let us introduce the group $\mathbb{R}_{\gamma}$ of all morphisms from $G_{\gamma}$ to $T$,

$$
\mathbb{R}_{\gamma}:=\operatorname{Hom}\left[G_{\gamma}, T\right]
$$

It is shown in [17] that the family of spaces $\mathbb{R}_{\gamma}$ is a well defined projective family, and that $\overline{\mathbb{R}}$ is precisely the projective limit of this family. Turning to measures, we know that measures in the projective limit $\overline{\mathbb{R}}$ are in one to one correspondence with (properly compatible) families of measures in the spaces of the projective family [18]. So, the Haar measure in $\overline{\mathbb{R}}$ is fully described by the family of measures that one obtains by push-forward, with respect to the natural projections

$$
p_{\gamma}: \overline{\mathbb{R}} \rightarrow \mathbb{R}_{\gamma}, \quad \bar{x} \mapsto \bar{x}_{\mid \gamma},
$$

where $\bar{x}_{\mid \gamma}$ denotes the restriction of $\bar{x}$ to the subgroup $G_{\gamma}$.

Now, since each $G_{\gamma}$ is freely generated by an independent set $\gamma$, each $\mathbb{R}_{\gamma}$ is homeomorphic to a $n$-torus $T^{n}$, with $n$ being the cardinality of the set $\gamma=\left\{k_{1}, \ldots, k_{n}\right\}$. Not surprisingly, one can easily check that the Haar measure on $\overline{\mathbb{R}}$ can be characterized as the (unique) measure such that the push-forward of the projections (5) gives precisely the Haar measure on the corresponding torus $T^{n}, \forall$ independent set $\gamma$.

\subsection{Support of the Haar measure}

We are now in position to give a characterization of the support of the measure $\mu_{0}$, following in part arguments presented in [11].

Proposition 1. The Haar measure $\mu_{0}$ on $\overline{\mathbb{R}}$ is supported on the set $W$ of all elements $\bar{x}$ which are everywhere discontinuous, as a map from $\mathbb{R}$ to $T$. 
To prove this, it is sufficient to show that the complement

$$
W^{\mathrm{c}}=\left\{\bar{x} \in \overline{\mathbb{R}} \mid \exists s_{0} \in \mathbb{R} \text { such that } \bar{x} \text { is continuous in } s_{0}\right\}
$$

is contained in a zero measure subset of $\overline{\mathbb{R}}$. Let us consider the sets

$$
\Theta_{U}=\{\bar{x} \in \overline{\mathbb{R}} \mid \exists I \text { such that } \bar{x}(I) \subset U\},
$$

where $I \subset \mathbb{R}$ is an open set and $U$ is a measurable subset of $T$ with $0<\mu_{H}(U)<1$. Here, $\mu_{H}(U)$ is the Haar measure of $U$. We will need first the following

Lemma 1. The set $\Theta_{U}$ is contained in a zero measure subset of $\overline{\mathbb{R}}$, for every $U \subset T$ such that $0<\mu_{H}(U)<1$.

To prove this lemma, let us start by recalling that the family of open balls

$$
B(q, 1 / m)=\{s \in[0,1]|| s-q \mid<1 / m\}
$$

with rational $q$ and integer $m$ constitutes a countable basis for the topology of $\mathbb{R}$, and therefore $\Theta_{U}$ is a countable union of sets of the form

$$
\Theta_{U, q, m}:=\{\bar{x} \in \overline{\mathbb{R}} \mid \bar{x}(B(q, 1 / m)) \subset U\}, \quad q \in \mathbb{Q}, \quad m \in \mathbb{N} .
$$

One can now construct zero measure sets containing each of the $\Theta_{U, q, m}$ as follows. For a given pair $(q, m)$, let us choose an infinite sequence $\left\{s_{i}\right\}_{i=1}^{\infty} \subset B(q, 1 / m)$ such that any of the finite subsets $\gamma_{N}:=\left\{s_{1}, \ldots, s_{N}\right\}$ is an independent set. This is possible, since given any finite independent set $\gamma$ one can always find another point $x_{0} \in B(q, 1 / m)$ such that $\gamma \cup x_{0}$ is again independent. Let us define the sets

$$
Z_{N}=\left\{\bar{x} \in \overline{\mathbb{R}} \mid \bar{x}\left(s_{i}\right) \in U, \forall s_{i} \in \gamma_{N}\right\}, \quad N \in \mathbb{N},
$$

which satisfy $Z_{N+1} \subset Z_{N}$. From the above mentioned relation between the measure $\mu_{0}$ and the measures obtained by means of the push-forward of the map (5), it follows that

$$
\mu_{0}\left(Z_{N}\right)=\left(\mu_{H}(U)\right)^{N}
$$

leading to

$$
\mu_{0}\left(\cap_{N=1}^{\infty} Z_{N}\right)=\lim _{N \rightarrow \infty}\left(\mu_{H}(U)\right)^{N}=0,
$$

by the $\sigma$-additivity of the measure and $\mu_{H}(U)<1$. On the other hand, $\Theta_{U, q, m} \subset Z_{N}, \forall N \in \mathbb{N}$, and therefore $\Theta_{U, q, m}$ is contained in the set $\cap_{N=1}^{\infty} Z_{N}$, of zero measure. It follows again from $\sigma$ additivity of $\mu_{0}$ that, $\forall U \subset T$ with $\mu_{H}(U)<1$, the set $\Theta_{U}$ is contained in a zero $\mu_{0}$-measure set.

Let us now conclude the proof of Proposition 1. Let $r$ be a real number, $0<r<1$, and consider a finite cover of $T$ by means of open sets $\left\{U_{i}\right\}_{i=1}^{k}$ such that $\mu_{H}\left(U_{i}\right)=r$, which is clearly possible. In fact, one can always take a finite subcovering of the covering consisting of neighbourhoods - of measure $r$ - of each and every point in $T$. Let $\bar{x}$ be any given element of $W^{\mathrm{c}}$ and $s_{0}$ a point of continuity. Let $i_{0}$ be such that $\bar{x}\left(s_{0}\right) \in U_{i_{0}}$. Continuity of $\bar{x}$ at $s_{0}$ implies that there exists a neighbourhood $I \ni s_{0}$ such that $\bar{x}(I) \subset U_{i_{0}}$. Therefore, every element of $W^{\mathrm{c}}$ belongs to one of the sets $\Theta_{U_{i}}$, i.e. $W^{\mathrm{c}} \subset \cup_{i=1}^{k} \Theta_{U_{i}}$. This concludes the prove of the Proposition, given Lemma 1. 


\subsection{Ergodic action}

Let us consider the multiplicative action of $\mathbb{R} \backslash\{0\}$ on $\mathbb{R},(\lambda, k) \mapsto \lambda k$, which obviously preserves the additive group structure, i.e. $\lambda\left(k_{1}+k_{2}\right)=\lambda k_{1}+\lambda k_{2}$. We therefore also have an action of $\mathbb{R} \backslash\{0\}$ on $\overline{\mathbb{R}},(\lambda, \bar{x}) \mapsto \lambda^{*} \bar{x}$, such that

$$
\lambda^{*} \bar{x}(k):=\bar{x}(\lambda k) .
$$

It is straightforward to check that the Haar measure $\mu_{0}$ is invariant under this action. By standard arguments (see $[2,14]$ ), it is sufficient to consider the integration of so-called cylindrical functions. In the present case these are essentially complex functions on a given $n$-torus, since they are defined as compositions of complex functions on the sets $\mathbb{R}_{\gamma}$ with the projections $p_{\gamma}(5)$. So, a general cylindrical function is of the form

$$
F(\bar{x})=f\left(\bar{x}\left(k_{1}\right), \ldots, \bar{x}\left(k_{n}\right)\right),
$$

where $\gamma=\left\{k_{1}, \ldots, k_{n}\right\}$ is an independent set and $f: T^{n} \rightarrow \mathbb{C}$ is an integrable function. Under the above action (6), such a cylindrical function transforms into

$$
\lambda^{*} F(\bar{x})=f\left(\bar{x}\left(\lambda k_{1}\right), \ldots, \bar{x}\left(\lambda k_{n}\right)\right) .
$$

So, the transformation amounts to switching from an independent set $\gamma$ to another independent set $\lambda \gamma:=\left\{\lambda k_{1}, \ldots, \lambda k_{n}\right\}$, of the same cardinality. Now

$$
\int_{\overline{\mathbb{R}}} F d \mu_{0}=\int_{T^{n}} f d\left(p_{\gamma_{*}} \mu_{0}\right) \quad \text { and } \quad \int_{\overline{\mathbb{R}}} \lambda^{*} F d \mu_{0}=\int_{T^{n}} f d\left(p_{\lambda \gamma_{*}} \mu_{0}\right),
$$

where $p_{\gamma_{*}} \mu_{0}$ and $p_{\lambda \gamma_{*}} \mu_{0}$ are given by push-forward of $\mu_{0}$ with respect to $p_{\gamma}$ and $p_{\lambda \gamma}$. (In fact, we are not showing explicitly the maps relating $\mathbb{R}_{\gamma}$ and $\mathbb{R}_{\lambda \gamma}$ to $T^{n}$. The argument is nevertheless rigorous, see, e.g., [2, 14] for totally similar arguments concerning the Ashtekar-Lewandowski measure.) But the measures $p_{\gamma_{*}} \mu_{0}$ and $p_{\lambda \gamma_{*}} \mu_{0}$ are actually the same, both being equal to the Haar measure on $T^{n}$, and we get

$$
\int_{\overline{\mathbb{R}}} F d \mu_{0}=\int_{\overline{\mathbb{R}}} \lambda^{*} F d \mu_{0} .
$$

Since this is true for every cylindrical function, one can conclude that the measure $\mu_{0}$ is invariant.

Proposition 2. The Haar measure on $\overline{\mathbb{R}}$ is ergodic with respect to the action of $\mathbb{R} \backslash\{0\}$ (6).

One can prove this result following lines of reasoning previously presented in [11]. We will use the well known fact that a measure is ergodic with respect to a group action if and only if the constant functions are the only (measurable) functions that remain invariant under the action of the group (see, e.g., [18]). In the present case this amounts to show that the only elements of $L^{2}\left(\overline{\mathbb{R}}, \mu_{0}\right)$ that remain invariant under the action (6) are the constant functions.

Let us first remind that the set of functions $\left\{F_{k}, k \in \mathbb{R}\right\}$ defined by $F_{k}(\bar{x})=\bar{x}(k)$ forms a complete orthonormal set on $L^{2}\left(\overline{\mathbb{R}}, \mu_{0}\right)$ (see, e.g., [17]). It is clear that under the action (induced from) (6), the functions $F_{k}$ transform amongst themselves:

$$
F_{k} \mapsto F_{\lambda k} .
$$

Let then $\psi$ be an element of $L^{2}\left(\overline{\mathbb{R}}, \mu_{0}\right)$. Since the set $\left\{F_{k}, k \in \mathbb{R}\right\}$ is an orthonormal basis, one can write $\psi$ in the form

$$
\psi=\sum_{k \in \mathbb{R}} c_{k} F_{k}
$$


with no more than countably many coefficients $c_{k}$ being nonzero. Given the transformation (7), one concludes that $\psi$ is invariant under the action (6) if and only if

$$
c_{k}=c_{\lambda k}, \quad \forall k \in \mathbb{R}, \quad \forall \lambda \in \mathbb{R} .
$$

Suppose there is $k \neq 0$ such that $c_{k} \neq 0$. Then it follows from (9) that in the sum (8) there is an infinite number of equal - nonzero - coefficients, which is clearly impossible. One then concludes that an invariant $\psi$ is proportional to the identity function $F_{0}$, i.e. is a constant.

\section{Acknowledgements}

I would like to thank José Mourão and also the anonymous referees for comments and corrections.

\section{References}

[1] Ashtekar A., Bojowald M., Lewandowski J., Mathematical structure of loop quantum cosmology, Adv. Theor. Math. Phys. 7 (2003), 233-268, gr-qc/0304074.

[2] Ashtekar A., Lewandowski J., Background independent quantum gravity: a status report, Classical Quantum Gravity 21 (2004), R53-R152, gr-qc/0404018.

[3] Ashtekar A., Singh P., Loop quantum cosmology: a status report, Classical Quantum Gravity 28 (2011), 213001, 122 pages, arXiv:1108.0893.

[4] Banerjee K., Calcagni G., Martín-Benito M., Introduction to loop quantum cosmology, SIGMA 8 (2012), 016, 73 pages, arXiv:1109.6801.

[5] Bojowald M., Loop quantum cosmology, Living Rev. Relativity 11 (2008), 4, 131 pages, gr-qc/0601085.

[6] Bojowald M., Mathematical structure of loop quantum cosmology: homogeneous models, SIGMA 9 (2013), 082, 43 pages, arXiv:1206.6088.

[7] Castelló Gomar L., Cortez J., Martín-de Blas D., Mena Marugán G.A., Velhinho J.M., Uniqueness of the Fock quantization of scalar fields in spatially flat cosmological spacetimes, J. Cosmol. Astropart. Phys. 2012 (2012), no. 11, 001, 21 pages, arXiv:1211.5176.

[8] Colella Ph., Lanford O.E., Appendix: Sample field behavior for the free Markov random field, in Constructive Quantum Field Theory (International School of Mathematical Physics, Erice (Sicily), 1973), Lecture Notes in Phys., Vol. 25, Editors G. Velo, A. Wightman, Springer-Verlag, Berlin - New York, 1973, 44-70.

[9] Glimm J., Jaffe A., Quantum physics. A functional integral point of view, 2nd ed., Springer-Verlag, New York, 1987.

[10] Marolf D., Mourão J.M., On the support of the Ashtekar-Lewandowski measure, Comm. Math. Phys. 170 (1995), 583-605, hep-th/9403112.

[11] Mourão J.M., Thiemann T., Velhinho J.M., Physical properties of quantum field theory measures, J. Math. Phys. 40 (1999), 2337-2353, hep-th/9711139.

[12] Reed M., Rosen L., Support properties of the free measure for Boson fields, Comm. Math. Phys. 36 (1974), $123-132$.

[13] Rivasseau V., From perturbative to constructive renormalization, Princeton Series in Physics, Princeton University Press, Princeton, NJ, 1991.

[14] Thiemann T., Modern canonical quantum general relativity, Cambridge Monographs on Mathematical Physics, Cambridge University Press, Cambridge, 2007.

[15] Velhinho J.M., Comments on the kinematical structure of loop quantum cosmology, Classical Quantum Gravity 21 (2004), L109-L113, gr-qc/0406008.

[16] Velhinho J.M., On the structure of the space of generalized connections, Int. J. Geom. Methods Mod. Phys. 1 (2004), 311-334, math-ph/0402060.

[17] Velhinho J.M., The quantum configuration space of loop quantum cosmology, Classical Quantum Gravity 24 (2007), 3745-3758, arXiv:0704.2397.

[18] Yamasaki Y., Measures on infinite-dimensional spaces, Series in Pure Mathematics, Vol. 5, World Sci. Publ. Co., Singapore, 1985. 